\title{
AMUSEVR: A Virtual Reality Authoring Environment for Immersive Mulsemedia Applications
}

\author{
FLÁVIO MIRANDA DE FARIAS, MídiaCom Lab, Fluminense Federal University, Brazil \\ DOUGLAS PAULO DE MATTOS, MídiaCom Lab, Fluminense Federal University, Brazil \\ DÉBORA C. MUCHALUAT-SAADE, MídiaCom Lab, Fluminense Federal University, Brazil
}

\begin{abstract}
To meet the demand for mulsemedia (multiple sensorial media) content production, numerous tools exist, among which many present the currently typical approach providing 2D content organized in timelines. However, when it comes to $360^{\circ}$ content where 3D spatial positioning is necessary, neither consensus on authoring methodology nor widely-adopted approaches to design exist. In order to fulfill this gap, we propose AMUSEVR, a virtual-reality environment for mulsemedia application development. In addition to supporting various media and sensory effect types, AMUSEVR allows authors to create and visualize content dynamically using VR technology. We evaluated AMUSEVR with an authoring experiment with users, using UEQ questionnaires, and obtained promising results.
\end{abstract}

CCS Concepts: $\bullet$ Information systems $\rightarrow$ Multimedia content creation; $\bullet$ Software and its engineering $\rightarrow$ Application specific development environments.

Additional Key Words and Phrases: $360^{\circ}$ authoring, VR environment, mulsemedia, event-based relations

\section{ACM Reference Format:}

Flávio Miranda de Farias, Douglas Paulo de Mattos, and Débora C. Muchaluat-Saade. 2021. AMUSEVR: A Virtual Reality Authoring Environment for Immersive Mulsemedia Applications. In SensoryX '21: Workshop on Multisensory Experiences, together with IMX 2021: ACM International Conference on Interactive Media Experiences. June 21-23, 2021. NY, USA. 4 pages.

\section{INTRODUCTION}

The evolution of multimedia applications has caused new approaches to emerge, such as Virtual Reality (VR) and 360-degree image and video, which provide the consumer a sense of being immersed within the media content. Therefore, they promise the public an offering of fresh narratives in communications, journalism and marketing, among other fields [7]. It should be noted that there are a few limitations to this technology in that the freedom of orientation introduces unpredictability in the user experience, as the audience risks losing essential elements or details, due to the forms of interaction with content, as well as the lack of experience of the content producers and the eventual audience [7].

In order to promote VR as a technology that offers an enhanced user experience [7], we need to provide tools which are capable of producing this kind of content to authors that are not programming experts. Available tools still use authoring techniques inherited from traditional technologies aimed at 2D media. Some presentations are built based on timelines [1], others on relationships between media [2]. However, the challenge persists of providing an authoring tool that allows manipulating $360^{\circ}$ media content in $3 \mathrm{D}$ space.

In addition, sensory effects may be specified to provide a greater immersive experience to users, generating 360-mulsemedia (multiple sensorial media) applications. Much of human communication relies on non-verbal cues and indicators. Therefore, we are interested in providing functionalities

Published in accordance with the terms of the Creative Commons Attribution 4.0 International Public License (CC BY 4.0). Permission to reproduce or distribute this work, in part or in whole, verbatim, adapted, or remixed, is granted without fee, provided that the appropriate credits are given to the original work, not implying any endorsement by the authors or by SBC.

(C) 2021 Brazilian Computing Society 
which allow stimulating human senses beyond sight and hearing for users to better interpret content offered by applications [7].

Focusing on filling this gap, this work proposes AMUSEVR, which is a virtual-reality environment for the creation of mulsemedia applications. AMUSEVR is an environment that provides immersion of the author, aiming at facilitating 360-mulsemedia application production as he/she can experience the same environment as the final user will, during authoring time [3].

The remainder of this paper is structured as follows. Section 2 discusses related work. Section 3 presents our proposal. Section 4 discusses experiment results and Section 5 presents final remarks.

\section{RELATED WORK}

In [6], Mendes et al. propose an authoring model that allows authors to design and create 360-degree interactive videos, focusing on their own declarative language based on XML. As 360-degree media was projected onto a spherical object surrounding the user, immersion was easily broken if the user happened to move out of the center of the sphere. In addition, the creative process is limited to direct editing in XML, to later be viewed through virtual-reality headset.

In [1], they propose an authoring tool with three different authoring interfaces (desktop, immersive, and tangible interface) for creating multisensory $360^{\circ}$ videos. This authoring tool allows the edition and visualization of $360^{\circ}$ video with the novelty of allowing to complement the video with multiple stimuli such as audio, haptics, and olfactory effects.

The work described in [4] has two main contributions: (1) it investigates existing highlighting methods for VR scene settings that are based on 3D models and explores their suitability for a $360^{\circ}$ video VR setting; (2) it proposes immersive authoring methods suitable for laymen to create highlights within a $360^{\circ}$ video VR application. However, although the tool is authored in real time, it is limited to creating menus and inserting highlights in video objects.

\section{AMUSEVR}

AMUSEVR is a virtual-reality environment for creating and experiencing fully immersive multimedia applications. It also interfaces with external hardware to provide sensory feedback to users. It was developed using the Unity engine, the HTC Vive virtual-reality headset and the Windows platform. For demonstration and usability tests, we used media content available at free websites ${ }^{1}$. AMUSEVR supports the MultiSEM model [2] by enabling various media object and sensory effect types synchronized by event-based relations.

The system provides a "Viewer Mode", where it is possible to import a saved project and thereby participate in interactive presentations. There is also an "Author Mode", where the author creates and edits the content in an immersive environment. In the Author Mode, as seen in Figure 1(a), the headset and controls allow the author to insert media objects, edit them by changing their properties individually or remove them from the project. For this purpose, we designed the scene menu to be positioned above a control device by default, while other control device is designed to point at the menus and further manipulate media objects.

A notable difference in the authoring methodology proposed and applied by AMUSEVR is that traditional applications work first by selecting the media object file, then editing or deleting them. However, in our solution, each media object presents different behavior and properties. For example, a 2D image can be selected through pointing, then edited or deleted. On the other hand, a 360-degree image must not be selected through pointing, as it could be selected unintentionally due to how the author's field of view is fully taken up by the 360-degree image. Therefore, the proposed authoring steps follow the first menu in Figure 1(a), depending on the type of media

\footnotetext{
${ }^{1}$ https://www.pexels.com/ and https://www.mettle.com/360vr-master-series-free-360-downloads-page/
} 


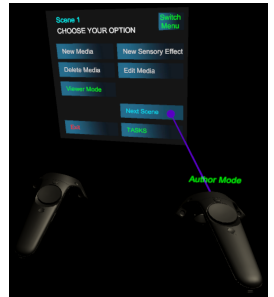

(a) Author Mode menu and controls.

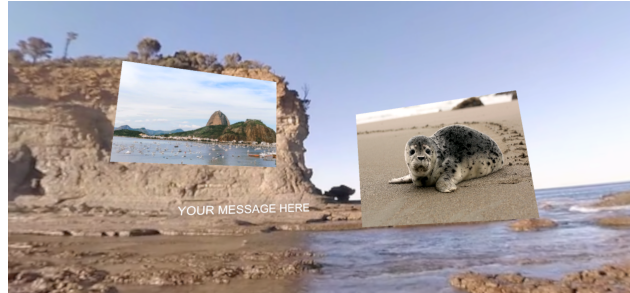

(b) 2D Media objects in a $360^{\circ}$ scene.

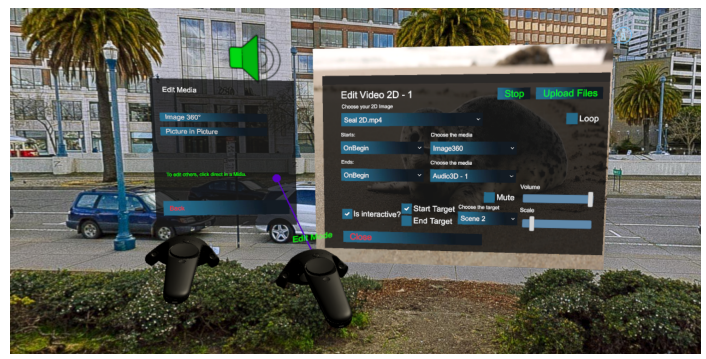

(c) Scene Editing Mode.

Fig. 1. Screenshots of AMUSEVR.

object. Figure 1(b) illustrates a very important aspect of the proposed 3D spatial concept, the ability to position $2 \mathrm{D}$ media objects anywhere in the $\mathrm{x}, \mathrm{y}$ and $\mathrm{z}$ axes within the $3 \mathrm{D}$ environment, simply with a drag-and-drop sequence.

The concept of scenes was proposed to determine the organization among media objects, where each scene is independent and has its own media objects, allowing the author to create multiple scenes with different themes, which allows for diverse scenarios in the same $360^{\circ}$ presentation. The user can navigate from one scene to another interacting with media objects in the previous scene.

Figure 1(c) shows a new scene Editing Mode in which we see a scenario composed of a 3D audio type media object, a 360-degree panoramic image and a 2D video with its editing properties being displayed. In this sample scene shown in Figure 1(c), we highlight how AMUSEVR provides editing methods, such as displaying or hiding media elements in the scene, as well as creating links to other scenes, thereby enabling transitions between scenes of the same project. This feature transforms projects from passive to interactive presentations, providing options to define interactive objects. These functions grant creative freedom and functionality within diverse applications where the user can, for example, move between the rooms of a virtual museum, or for 360 degree films, switch between spoken or subtitled languages, or even start rendering a sensory effect.

Another way to control the presentation of media objects in the scene is through the event-based relationship paradigm, in which it is possible to associate the beginning and end of the presentation of a media object to another media object. Notice that in Figure 1(c), the "Starts" menu defines a start relationship with media object "Image360" through the command "OnBegin". That is, the object being edited, called "Video 2D - 1", will only start when object "Image 360" is started. For the same "Video 2D - 1" object, we have another property called "Ends" related to the beginning ("OnBegin") of the "Audio3D - 1" object. Thus, "Video 2D - 1" will only end when object "Audio3D - 1" begins. Therefore, it is possible for the author to schedule the presentation of objects in the scene in an event-based manner and to remove them from the scene automatically as needed. 


\section{USER EXPERIMENTS}

Tests have been carried out with 5 users, men and women aged between 23 to 37 years old, with education levels ranging from under-graduate to post-graduate. Only one had not used VR equipment previously, while another had not had experience with multimedia authoring systems. Each one decided to sit or stand during the experience, with a supervisor monitoring the test.

All participants were given time to acquaint themselves with the environment and tools used (5 minutes on average), after which, each of them started the tasks and had their results recorded. They have to create an interactive 360-mulsemedia presentation of a tourism showcase of the city of Rio de Janeiro. To guide the user doing the test, there was a reference table in the control menu describing the tasks to be performed. After the tests, they were asked to fill out a survey which covered the User Experience Questionnaire - UEQ [5].

Those four users who had previous experience with authoring tools completed the test in 6 to 7 minutes, while the one who had no previous experience also succeeded, but required 14 minutes in total, indicating that even non-experts users can use the system, with prior experience being the primary difference in the time taken to complete the tasks.

UEQ has scores ranging from -3 (worst result) to 3 (best result) and has 8 questions. The results indicate half of the questions pointing to "Pragmatic Quality" (average around 2.0) and the rest to "Hedonic Quality" (around 2.5), which classifies the usability of the system as excellent according to the UEQ evaluative requirements [5].

\section{CONCLUSIONS}

This paper proposed AMUSEVR, a virtual-reality environment for interactive mulsemedia application development. User practical experiments reported viable results, where all users completed their tasks of creating a $360^{\circ}$ interactive mulsemedia presentation.

An important future work is the definition of an XML language for authoring $360^{\circ}$ interactive mulsemedia content in order to facilitate project interchange among AMUSEVR and other authoring tools. We also expect to run further user experiments to refine our solution over time. AMUSEVR is currently limited to the Vive environment, we intend to extend it to Oculus Quest. Another future work is to extend UI-control compatibility with Google Cardboard.

\section{ACKNOWLEDGMENTS}

This work was partially funded by CAPES, CNPq and FAPERJ.

\section{REFERENCES}

[1] H Coelho, M Melo, L Barbosa, J Martins, M S Teixeira, and M Bessa. 2019. Authoring tools for creating 360 multisensory videos-Evaluation of different interfaces. Expert Systems (2019). https://doi.org/10.1111/exsy.12418

[2] D P de Mattos, D C Muchaluat-Saade, and G Ghinea. 2020. An Approach for Authoring Mulsemedia Documents Based on Events. In 2020 International Conference on Computing, Networking and Communications (ICNC). IEEE, 273-277.

[3] Hannes Fassold and Barnabas Takacs. 2019. Towards Automatic Cinematography and Annotation for $360^{\circ}$ Video. In Proceedings of the 2019 ACM International Conference on Interactive Experiences for TV and Online Video. 157-166.

[4] Robin Horst, Savina Diez, and Ralf Dörner. 2019. Highlighting Techniques for $360^{\circ}$ Video Virtual Reality and Their Immersive Authoring. In Lecture Notes in Computer Science (including subseries Lecture Notes in Artificial Intelligence and Lecture Notes in Bioinformatics), Vol. 11844 LNCS. Springer, 515-526. https://doi.org/10.1007/978-3-030-33720-9_40

[5] Bettina Laugwitz, Theo Held, and Martin Schrepp. 2008. Construction and Evaluation of a User Experience Questionnaire. HCI and Usability for Education and Work, 4th Symposium of the Workgroup Human-Computer Interaction and Usability Engineering of the Austrian Computer Society 5298 (2008), 63-76. https://doi.org/10.1007/978-3-540-89350-9_6

[6] Paulo R C Mendes, Álan L V Guedes, Daniel De S Moraes, Roberto G A Azevedo, and Sérgio Colcher. 2020. An Authoring Model for Interactive 360 Videos. 2020 IEEE International Conference on Multimedia \& Expo Workshops (ICMEW) (2020).

[7] Mel Slater and Maria V. Sanchez-Vives. 2016. Enhancing our lives with immersive virtual reality. Frontiers Robotics AI 3 , DEC (dec 2016), 74. https://doi.org/10.3389/frobt.2016.00074 